\title{
Publisher's Note: Interpenetrating graphene networks: Three-dimensional node-line semimetals with massive negative linear compressibilities [Phys. Rev. B 94, 245422 (2016)]
}

\author{
Yangzheng Lin, Zhisheng Zhao, Timothy A. Strobel, and R. E. Cohen \\ (Received 10 January 2017; published 17 January 2017)
}

DOI: 10.1103/PhysRevB.95.039903

This paper was published online on 19 December 2016 with an incorrect version of Fig. 2 and an error in the caption of Fig. 10. The second line of the caption to Fig. 10 should read as "... two-dimensional electronic band dispersion in Z11. (a) The Fermi ...." The caption has been corrected and Fig. 2 has been replaced as of 27 December 2016. The text and figure are correct in the printed version of the journal. 